\title{
Stellar populations in late-type spirals observed with SAURON
}

\author{
Katia Ganda ${ }^{1}$, Reynier F. Peletier ${ }^{1}$, \\ Jesús Falcón-Barroso ${ }^{2,3}$ and Richard M. McDermid ${ }^{2}$ \\ ${ }^{1}$ Kapteyn Astronomical Institute, P.O. BOX 800, 9700AV, Groningen, The Netherlands \\ email: katia@astro.rug.nl \\ ${ }^{2}$ Leiden Observatory, Postbus 9513, 2300 RA Leiden, The Netherlands \\ ${ }^{3}$ European Space Agency, ESTEC, Postbus 299, 2200 AG Noordwijk, The Netherlands
}

\begin{abstract}
We present results on the stellar populations of a sample of 18 late-type spirals, based on data acquired with the integral-field spectrograph SAURON at the WHT. We present the two-dimensional line-strength maps, the central line indices, and estimate the star formation time-scale. In an exponentially declining SFR scenario, we find a trend between the time-scale $\tau$ and the central velocity dispersion: more massive galaxies show shorter burst durations. A detailed study on these data is published by Ganda et al. (2007).
\end{abstract}

Keywords. galaxies: individual (NGC2964), galaxies: evolution, galaxies: spiral

\section{Introduction}

We are carrying out a project on the kinematics and stellar populations in late-type spiral galaxies, objects whose stellar component has not been studied in detail yet, due to their low surface brightness and contamination by dust and gas. We focus on a sample of 18 galaxies (morphological type $\mathrm{Sb}$ to $\mathrm{Sd}$ ) that we observed with the integral-field spectrograph SAURON, that allows measurement of the Lick-indices $\mathrm{H} \beta$, Fe5015 and $\mathrm{Mg} b$ on a two-dimensional field of view of $(41 \times 33) \operatorname{arcsec}^{2}$. We present here example maps of the indices, the inferred stellar population parameters and our results on the star formation history.

\section{Stellar populations and star-formation history of late-type spirals}

The sample selection, data reduction and the extracted kinematics of stars and gas are described in detail by Ganda et al. (2006). Continuing the work presented there, we cleaned the spectra from emission lines and computed the line-strength indices. In Figure 1 we show the line index maps for NGC2964 (Sbc). Maps for the whole sample are presented and commented by Ganda et al. (2007).

In Figure 2 (left panel) we plot the central line indices of our galaxies (dots) in the plane $([\mathrm{MgFe} 50], H \beta)$; we overplot the SSP model grid from Thomas et al. (2003) (solid lines) and the E/S0 and Sa samples (respectively, asterisks and triangles) observed with SAURON and discussed by Kuntschner et al. (2006) and Peletier et al. (2007). This diagram, that shows ages independently of abundance ratios, confirms that the centres of late-type spirals are in general younger and more metal-poor than E/S0 galaxies. It also shows a smaller spread in age and metallicity, compared to Sa galaxies.

We also addressed the star formation history of our galaxies. We calculated the spectral evolution of SSP models for various metallicities and Chabrier IMF, using tools provided online by Bruzual \& Charlot (2003) and assuming an exponentially declining 

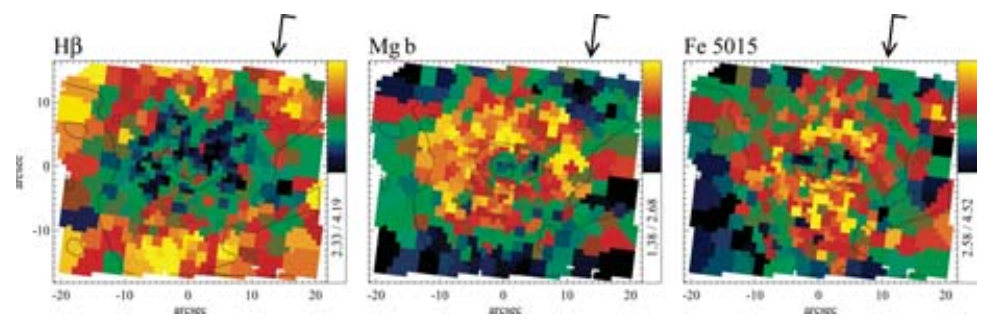

Figure 1. NGC2964: maps of the Lick indices $\mathrm{H} \beta, \mathrm{Mg} b$ and Fe5015 (equivalent widths, in $\AA$ ) for NGC2964. The direction of North and East are indicated by the arrow. Overplotted are the isophotal contours.
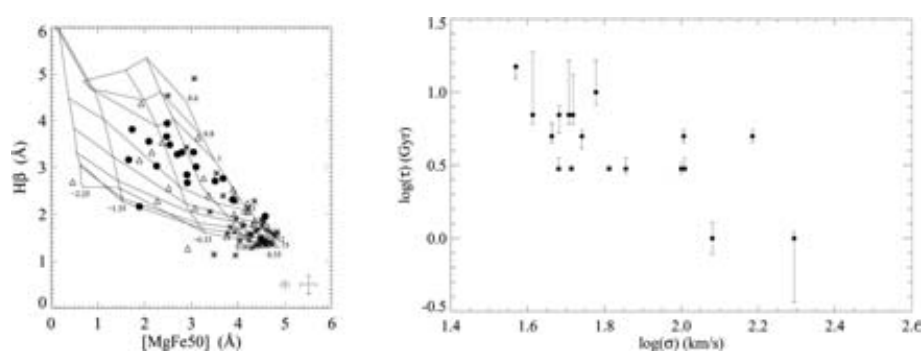

Figure 2. Left panel: $\mathrm{H} \beta$ against [MgFe50] ; dots: late-type spirals; asterisks: E/S0; triangles: Sa galaxies (all SAURON data); solid lines: SSP model grid from Thomas et al. (2003), with age increasing from top to bottom and metallicity from left to right. Right panel: $\tau$ against central velocity dispersion for the 18 late-type spirals, in a log-log scale.

star formation rate: $\psi(t)=1 M \odot \tau^{-1} \exp (-t / \tau)$, where $\psi(t)$ and $\tau$ are the SFR and the e-folding time-scale. $\tau$ parametrizes a smooth transition from SSP-like to constant SFR (small $\tau \approx$ SSP, large $\tau \approx$ constant SFR). Interpreting the indices variations as variations in the SFR time-scale, we can get an estimate of $\tau$ by comparing our observed line-strength indices with those from the evolved models. In Figure 2 (right panel) we plot $\tau$ against velocity dispersion, for age of the evolved models fixed to $10 \mathrm{Gyr}$, and find that bigger galaxies tend to have shorter SFR time-scales.

\section{Conclusions}

We have presented line-strength maps for late-type spirals and estimated the timescale of star formation. We found that larger galaxies can be approximated with SSPs better than smaller ones. We compared their stellar populations with those of earlier types: late-types are generally younger and more metal-poor.

\section{References}

Bruzual, C. \& Charlot, S. 2003, MNRAS, 344, 1000

Ganda, K., Falcón-Barroso J., \& Peletier R. F., et al. 2006, MNRAS, 367, 46

Ganda, K., et al. 2007, MNRAS, 380, 506

Kuntschner, H., Emsellem, E., \& Bacon, R., et al. 2006, MNRAS, 369, 497

Peletier, R. F., et al. 2007, MNRAS, 379, 445

Thomas, D., Maraston, C., \& Bender, R. 2003, MNRAS, 339, 897 